\title{
Analytical solution for viscous incompressible Stokes flow in a spherical shell
}

\author{
Cedric Thieulot \\ Department of Earth Sciences, Utrecht University, Utrecht, the Netherlands \\ Correspondence to: Cedric Thieulot (c.thieulot@uu.nl)
}

Received: 6 July 2017 - Discussion started: 27 July 2017

Revised: 19 September 2017 - Accepted: 27 September 2017 - Published: 24 November 2017

\begin{abstract}
I present a new family of analytical flow solutions to the incompressible Stokes equation in a spherical shell. The velocity is tangential to both inner and outer boundaries, the viscosity is radial and of the power-law type, and the solution has been designed so that the expressions for velocity, pressure, and body force are simple polynomials and therefore simple to implement in (geodynamics) codes. Various flow average values, e.g., the root mean square velocity, are analytically computed. This forms the basis of a numerical benchmark for convection codes and I have implemented it in two finite-element codes: ASPECT and ELEFANT. I report error convergence rates for velocity and pressure.
\end{abstract}

\section{Introduction}

The mantle is a very complex region characterized by large variations in temperature, viscosity, composition (Schubert et al., 2001; van Keken et al., 2002), phase changes, melting, and anisotropic structures as revealed by seismic imaging (Lay and Garnero, 2011). As a consequence, the numerical modeling of mantle convection has grown in complexity (e.g., Tackley, 2012; van Heck et al., 2016; Dannberg and Heister, 2016) with the advent of ever more refined modeling techniques and powerful computers. It has also been recognized that the mantle exerts a primary control on the evolution of tectonic plates and that both should be simulated together if one is to build a fully dynamic Earth model (e.g., van Hinsbergen et al., 2011; Bull et al., 2014; Bower et al., 2015; Crameri and Tackley, 2016).

Many codes have been developed in the last 30 years (Machetel et al., 1986; Glatzmaier, 1988; Bercovici et al., 1989; Zhang and Christensen, 1993; Zhang and Yuen, 1995; Rat- cliff et al., 1996; Iwase, 1996; Zhong et al., 2000; Tabata and Suzuki, 2000; Richards et al., 2001; Kageyama and Sato, 2004; Yoshida and Kageyama, 2004; Choblet et al., 2007; Tackley, 2008; Davies et al., 2013; Kronbichler et al., 2012; Burstedde et al., 2013), and spherical shell numerical benchmarks have been carried out (Zhong et al., 2000; Stemmer et al., 2006; Zhong et al., 2008; Arrial et al., 2014). Semianalytical Stokes flow solutions derived via propagator matrix methods have also been proposed in the past (Busse, 1975; Busse and Riahi, 1982; Hager and O'Connell, 1981; Richards and Hager, 1984), while Tosi and Martinec (2007) derived a semi-analytical solution in the case of two eccentrically nested spheres. However, semi-analytical solutions present a major drawback: the solution is given as a function of spherical harmonic expansions, which are based on infinite sums and which can prove to be cumbersome to manipulate and/or implement.

While inter-code comparisons are useful for problems without an analytical solution (e.g., Arrial et al., 2014; Tosi et al., 2015), such benchmark studies rely on the comparisons between a handful of scalar values (e.g., root mean square velocity, Nusselt number), which account for the global character of the solution at steady state but do not lend themselves to error convergence measurements. Fully analytical solutions have also been recently proposed, attempting to represent a mid-ocean ridge (Burstedde et al., 2013) or being more abstract in nature (Zhong, 1996; Popov et al., 2014; Blinova et al., 2016). Actually, any analytical solution to the Stokes equation in three dimensions could be used in a spherical shell provided that the velocity is appropriately applied on the inner and outer boundaries (e.g., Burstedde et al., 2013), but such solutions usually do not satisfy the condition $\boldsymbol{v} \cdot \boldsymbol{n}=0$ on the inner and outer boundaries; i.e., there is flow 
through the boundaries. Not only does the presence of a material flow through the boundaries make the flow not Earthlike, but it also precludes its use for particle-in-cell advection benchmarking (particles would leave and enter the domain).

I propose a new analytical solution for viscous incompressible Stokes flow in a spherical shell. It has been designed with three constraints in mind: (1) the boundary conditions, buoyancy forces, and viscosity fields should be simple to implement and exact; (2) the solution should also be smooth and simple enough to be usable; (3) and it should satisfy tangential slip boundary conditions on both surfaces. I present in Sect. 2 the simplified Stokes equation in spherical coordinates under these flow assumptions and outline the procedure to arrive at the analytical solution for pressure and velocity in Sect. 3, for both constant and depth-dependent viscosity profiles. I compute in Sect. 4 the exact analytical values for the root mean square velocity and various other averages. In Sect. 5 the two numerical codes are introduced and the results obtained with these are shown. Finally, these results are discussed in Sect. 6.

\section{The Stokes equations in spherical coordinates}

The domain is a spherical shell parameterized by its inner radius $R_{1}$ and outer radius $R_{2}$. For an incompressible fluid, the Stokes flow equations are given by

$\nabla \cdot v=0$

$\nabla \cdot \sigma+\rho \boldsymbol{g}=\mathbf{0}$

where $\boldsymbol{v}$ is the velocity vector, $\rho$ is the mass density, $\boldsymbol{g}$ is the gravitational acceleration vector, and $\sigma$ is the full stress tensor, which can be split:

$\boldsymbol{\sigma}=-p \mathbf{1}+\boldsymbol{s}$

where $p$ is the pressure, $\mathbf{1}$ is the unit tensor, and $\boldsymbol{s}$ is the deviatoric stress tensor. Equation (2) then becomes

$-\nabla p+\nabla \cdot s+\rho g=0$.

In spherical coordinates, Eqs. (1) and (4) become

$$
\begin{aligned}
& \frac{1}{r^{2}} \frac{\partial}{\partial r}\left(r^{2} v_{r}\right)+\frac{1}{r \sin \theta} \frac{\partial}{\partial \theta}\left(v_{\theta} \sin \theta\right)+\frac{1}{r \sin \theta} \frac{\partial v_{\phi}}{\partial \phi}=0 \\
& -\frac{\partial p}{\partial r}+\frac{\partial s_{r r}}{\partial r}+\frac{1}{r} \frac{\partial s_{\theta r}}{\partial \theta}+\frac{1}{r \sin \theta} \frac{\partial s_{\phi r}}{\partial \phi} \\
& +\frac{2 s_{r r}-s_{\theta \theta}-s_{\phi \phi}}{r}+\frac{s_{\theta r} \cot \theta}{r}+\rho g_{r}=0 \\
& -\frac{1}{r} \frac{\partial p}{\partial \theta}+\frac{\partial s_{r \theta}}{\partial r}+\frac{1}{r} \frac{\partial s_{\theta \theta}}{\partial \theta}+\frac{1}{r \sin \theta} \frac{\partial s_{\phi \theta}}{\partial \phi} \\
& +\frac{3 s_{\theta r}+\left(s_{\theta \theta}-s_{\phi \phi}\right) \cot \theta}{r}+\rho g_{\theta}=0
\end{aligned}
$$

$$
\begin{aligned}
& -\frac{1}{r \sin \theta} \frac{\partial p}{\partial \phi}+\frac{\partial s_{r \phi}}{\partial r}+\frac{1}{r} \frac{\partial s_{\theta \phi}}{\partial \theta}+\frac{1}{r \sin \theta} \frac{\partial s_{\phi \phi}}{\partial \phi} \\
& +\frac{3 s_{r \phi}+2 s_{\phi \theta} \cot \theta}{r}+\rho g_{\phi}=0 .
\end{aligned}
$$

In this work, the following spherical coordinate conventions are used: $r$ is the radial distance, $\theta \in[0, \pi]$ is the polar angle, and $\phi \in[0,2 \pi]$ is the azimuthal angle. In the case of an incompressible fluid, the deviatoric stress is simply

$\boldsymbol{s}=2 \mu \dot{\boldsymbol{\epsilon}}$,

where $\mu$ is the dynamic viscosity, which can depend on space coordinates, and $\dot{\boldsymbol{\epsilon}}$ is the (deviatoric) strain rate tensor:

$\dot{\boldsymbol{\epsilon}}=\frac{1}{2}\left(\nabla \boldsymbol{v}+\nabla \boldsymbol{v}^{T}\right)$.

In spherical coordinates, the components of the deviatoric stress tensor are given by

$$
\begin{aligned}
& s_{r r}=2 \mu\left(\frac{\partial v_{r}}{\partial r}\right), \\
& s_{\theta \theta}=2 \mu\left(\frac{1}{r} \frac{\partial v_{\theta}}{\partial \theta}+\frac{v_{r}}{r}\right), \\
& s_{\phi \phi}=2 \mu\left(\frac{1}{r \sin \theta} \frac{\partial v_{\phi}}{\partial \phi}+\frac{v_{r}}{r}+\frac{v_{\theta}}{r} \cot \theta\right), \\
& s_{r \theta}=s_{\theta r}=\mu\left(\frac{\partial v_{\theta}}{\partial r}-\frac{v_{\theta}}{r}+\frac{1}{r} \frac{\partial v_{r}}{\partial \theta}\right), \\
& s_{r \phi}=s_{\phi r}=\mu\left(\frac{1}{r \sin \theta} \frac{\partial v_{r}}{\partial \phi}+\frac{\partial v_{\phi}}{\partial r}-\frac{v_{\phi}}{r}\right), \\
& s_{\theta \phi}=s_{\phi \theta}=\mu\left(\frac{1}{r} \frac{\partial v_{\phi}}{\partial \theta}-\frac{\cot \theta v_{\phi}}{r}+\frac{1}{r \sin \theta} \frac{\partial v_{\theta}}{\partial \phi}\right) .
\end{aligned}
$$

Equations (5)-(8) supplemented by Eqs. (11)-(16) form a closed set of PDEs that can be solved given an appropriate set of boundary conditions.

\subsection{Assumptions on the flow}

In order to derive an analytical solution for the flow velocity and pressure in the domain, the following assumptions are made.

- All quantities $v_{r}, v_{\theta}, v_{\phi}, p, \rho$, and $\mu$ are independent of the azimuthal angle $\phi$. As a consequence, all the terms containing partial derivatives with respect to $\phi$ can be discarded. This is one of the most stringent limitations in this work since it implies rotational symmetry with respect to the vertical axis.

- The polar and azimuthal components of the velocity are equal and of the form

$$
v_{\theta}(r, \theta)=v_{\phi}(r, \theta)=f(r) \sin \theta .
$$


- The radial component of the velocity is null on the inside $r=R_{1}$ and outside $r=R_{2}$ of the domain, thereby ensuring a tangential flow on the boundaries, i.e.,

$v_{r}\left(R_{1}, \theta\right)=v_{r}\left(R_{2}, \theta\right)=0$.

- The viscosity is a function of the radial distance only and takes the form

$\mu(r)=\mu_{0} r^{m+1}$,

where $m$ is an integer (positive or negative). Note that $m=-1$ yields a constant viscosity.

- The gravity vector is set to $\boldsymbol{g}=-\boldsymbol{e}_{r}$ and is therefore of unit norm, i.e., $|\boldsymbol{g}|=1$.

\subsubsection{The set of simplified partial differential equations}

Under the above assumptions, it is easy to show that the Stokes equation in spherical coordinates is given by

$$
\begin{aligned}
& \frac{1}{r^{2}} \frac{\partial}{\partial r}\left(r^{2} v_{r}\right)+\frac{1}{r \sin \theta} \frac{\partial}{\partial \theta}\left(v_{\theta} \sin \theta\right)=0, \\
& -\frac{\partial p}{\partial r}+\rho(r, \theta)+\mu(r)\left(\Delta v_{r}-\frac{2 v_{r}}{r^{2}}\right. \\
& \left.\quad-\frac{2}{r^{2}} \frac{\partial v_{\theta}}{\partial \theta}-\frac{2 v_{\theta} \cot \theta}{r^{2}}\right)+2 \mu^{\prime}(r) \frac{\partial v_{r}}{\partial r}=0, \\
& -\frac{1}{r} \frac{\partial p}{\partial \theta}+\mu(r)\left(\Delta v_{\theta}+\frac{2}{r^{2}} \frac{\partial v_{r}}{\partial \theta}-\frac{v_{\theta}}{r^{2} \sin ^{2} \theta}\right) \\
& \quad+\mu^{\prime}(r)\left(\frac{\partial v_{\theta}}{\partial r}-\frac{v_{\theta}}{r}+\frac{1}{r} \frac{\partial v_{r}}{\partial \theta}\right)=0, \\
& \mu(r)\left(\Delta v_{\phi}-\frac{v_{\phi}}{r^{2} \sin ^{2} \theta}\right)+\mu^{\prime}(r)\left(\frac{\partial v_{\phi}}{\partial r}-\frac{v_{\phi}}{r}\right)=0,
\end{aligned}
$$

where $\Delta$ is the Laplacian operator:

$$
\begin{aligned}
\Delta= & \frac{1}{r^{2}} \frac{\partial}{\partial r}\left(r^{2} \frac{\partial}{\partial r}\right)+\frac{1}{r^{2} \sin \theta} \frac{\partial}{\partial \theta}\left(\sin \theta \frac{\partial}{\partial \theta}\right) \\
& +\frac{1}{r^{2} \sin ^{2} \theta} \frac{\partial^{2}}{\partial \phi^{2}} .
\end{aligned}
$$

\section{Derivation of the analytical solution of the Stokes equations}

My goal is to determine the $f(r)$ function in the context of all the assumptions made about the flow nature. The following four steps will then be taken:

1. use the continuity Eq. (20) to arrive at $v_{r}(r, \theta)=$ $g(r) \cos \theta$;
2. use the $\theta$ component of Stokes Eq. (22) to arrive at $p(r, \theta)=h(r) \cos \theta$;

3. use the $\phi$ component of Stokes Eq. (23) to arrive at $f(r)$ and $g(r)$ using boundary conditions on $v_{r}$;

4. and use the $r$ component of Stokes Eq. (21) to arrive at the density $\rho(r, \theta)$.

\subsection{Using the continuity equation to arrive at $v_{r}$}

Inserting Eq. (17) into Eq. (20) yields

$v_{r}(r, \theta)=g(r) \cos \theta$,

$g(r)=-\frac{2}{r^{2}} \int r f(r) \mathrm{d} r$,

where the integration constant has been set to zero for simplicity.

I then proceed to compute the Laplacian of each velocity component using Eq. (24), and once again all partial derivatives with respect to $\phi$ are neglected:

$$
\begin{aligned}
& \Delta v_{r}=\left[g^{\prime \prime}+\frac{2 g^{\prime}}{r}-2 \frac{g}{r^{2}}\right] \cos \theta, \\
& \Delta v_{\theta}=\left(f^{\prime \prime}+\frac{2 f^{\prime}}{r}\right) \sin \theta+\frac{f}{r^{2} \sin \theta}\left(\cos ^{2} \theta-\sin ^{2} \theta\right), \\
& \Delta v_{\phi}=\left(f^{\prime \prime}+\frac{2 f^{\prime}}{r}\right) \sin \theta+\frac{f}{r^{2} \sin \theta}\left(\cos ^{2} \theta-\sin ^{2} \theta\right) .
\end{aligned}
$$

\subsection{Using the $\theta$ component of Stokes equations to arrive at the pressure $p$}

Inserting Eqs. (17) and (25) into Eq. (22) yields

$\frac{\partial p}{\partial \theta}=-h(r) \sin \theta$

with

$h(r)=-\mu\left(2 f^{\prime}+r f^{\prime \prime}\right)+\frac{2 \mu+r \mu^{\prime}}{r}(f+g)-r \mu^{\prime} f^{\prime}$

so that

$p(r, \theta)=h(r) \cos \theta$,

where the integration constant has once again been omitted for simplicity.

\subsection{Using the $\phi$ component of Stokes equations to arrive at $f(r)$ and $g(r)$}

Inserting Eqs. (17) and (25) into Eq. (23) yields

$\mu\left(r^{2} f^{\prime \prime}+2 r f^{\prime}-2 f\right)+r \mu^{\prime}\left(r f^{\prime}-f\right)=0$.

I now make use of Eq. (19) so that Eq. (33) becomes

$r^{m+1}\left[r^{2} f^{\prime \prime}+(3+m) r f^{\prime}-(3+m) f\right]=0$. 
This equation has to hold for all $r$ values so that $f(r)$ is the solution of the second-order ODE:

$r^{2} f^{\prime \prime}+(3+m) r f^{\prime}-(3+m) f=0$.

I postulate that the solution is of the form $f(r)=r^{a}$, which yields

$[a+(m+3)](a-1) f(r)=0$.

The only two acceptable values for $a$ are the roots of this second-order polynomial, i.e., $a=1$ or $a=-(m+3)$. The general solution of the ODE is then

$f(r)=\alpha r^{-(m+3)}+\beta r$,

where $\alpha$ and $\beta$ are two constants yet to be determined. Having obtained $f(r)$, one can now compute $g(r)$. However, as will become obvious, one must make a distinction between $m=-1$ and $m \neq-1$.

Note that the case $m=-3$ should be considered separately since Eq. (35) then becomes $f^{\prime \prime}(r)=0$ and yields a solution $f(r)=\beta r$.

\subsubsection{Case $m=-1$}

In this case the function $f(r)$ is

$f(r)=\frac{\alpha}{r^{2}}+\beta r$,

and from Eq. (26) one obtains

$g(r)=-\frac{2}{r^{2}}\left(\alpha \ln r+\frac{\beta}{3} r^{3}+\gamma\right)$,

where $\gamma \neq 0$ is a constant. From Eqs. (18) and (25) it follows that $g\left(R_{1}\right)=g\left(R_{2}\right)=0$, which yields

$\alpha=-\gamma \frac{R_{2}^{3}-R_{1}^{3}}{R_{2}^{3} \ln R_{1}-R_{1}^{3} \ln R_{2}}$,

$\beta=-3 \gamma \frac{\ln R_{2}-\ln R_{1}}{R_{1}^{3} \ln R_{2}-R_{2}^{3} \ln R_{1}}$.

\subsubsection{Case $m \neq-1$}

In this case the function $g(r)$ takes the form

$g(r)=-\frac{2}{r^{2}}\left(-\frac{\alpha}{m+1} r^{-(m+1)}+\frac{\beta}{3} r^{3}+\gamma\right)$,

and the boundary conditions impose

$\alpha=\gamma(m+1) \frac{R_{1}^{-3}-R_{2}^{-3}}{R_{1}^{-(m+4)}-R_{2}^{-(m+4)}}$,

$\beta=-3 \gamma \frac{R_{1}^{m+1}-R_{2}^{m+1}}{R_{1}^{m+4}-R_{2}^{m+4}}$.

Note that this imposes $m \neq-4$.

\subsection{Using the $r$ component of Stokes equations to arrive at density $\rho$}

Equation (21) contains the term $\partial p / \partial r=\partial h / \partial r \cos \theta$, which needs to be addressed beforehand:

$$
\begin{aligned}
\frac{\partial h}{\partial r}= & -\mu^{\prime}\left(2 f^{\prime}+r f^{\prime \prime}\right)-\mu\left(3 f^{\prime \prime}+r f^{\prime \prime \prime}\right) \\
& +\frac{r^{2} \mu^{\prime \prime}+2 r \mu^{\prime}-2 \mu}{r^{2}}(f+g) \\
& +\frac{2 \mu+r \mu^{\prime}}{r}\left(f^{\prime}+g^{\prime}\right)-\mu^{\prime} f^{\prime}-r \mu^{\prime \prime} f^{\prime}-r \mu^{\prime} f^{\prime \prime} .
\end{aligned}
$$

\subsubsection{Case $m=-1$}

In this case, $\mu^{\prime}=0$ and $\mu^{\prime \prime}=0$ so that $\partial h / \partial r \cos \theta$ simplifies to

$\frac{\partial h}{\partial r}=-\left(3 f^{\prime \prime}+r f^{\prime \prime \prime}\right)+\frac{2}{r}\left(f^{\prime}+g^{\prime}\right)-\frac{2}{r^{2}}(f+g)$,

and Eq. (21) becomes

$-\frac{\partial h}{\partial r}+\frac{\rho(r, \theta)}{\cos \theta}+\frac{\Delta v_{r}}{\cos \theta}-\frac{1}{r^{2}}(2 g+4 f)=0$.

I postulate that

$\rho(r, \theta)=\mathcal{F}(r) \cos \theta$,

and by using Eq. (27), the radial function $\mathcal{F}$ is given by

$\mathcal{F}(r)=-r f^{\prime \prime \prime}-3 f^{\prime \prime}+2 \frac{f^{\prime}}{r}-g^{\prime \prime}+\frac{2}{r^{2}}(f+g)$.

Inserting the radial functions $f(r)$ and $g(r)$ given in Eqs. (38) and (39) into Eq. (49) yields

$\rho(r, \theta)=\left(\frac{\alpha}{r^{4}}(8 \ln r-6)+\frac{8 \beta}{3 r}+8 \frac{\gamma}{r^{4}}\right) \cos \theta$.

\subsubsection{Case $m \neq-1$}

Using Eq. (19) leads to

$$
\begin{aligned}
\frac{1}{r^{m}} \frac{\partial h}{\partial r}= & -r^{2} f^{\prime \prime \prime}-[2 m+5] r f^{\prime \prime}-m(m+3) f^{\prime} \\
& +m(m+3)(f+g) / r+(m+3) g^{\prime} .
\end{aligned}
$$

Then Eq. (21) becomes

$$
\begin{aligned}
- & \frac{\partial h}{\partial r} \cos \theta+\rho(r, \theta)+\mu(r)\left[\Delta v_{r}-\frac{\cos \theta}{r^{2}}(2 g+4 f)\right] \\
& +\mu^{\prime}(r) g^{\prime}(r) \cos \theta=0 .
\end{aligned}
$$

I postulate here that

$\rho(r, \theta)=r^{m} \mathcal{F}(r) \cos \theta$ 
and arrive at

$$
\begin{gathered}
\mathcal{F}(r)=\frac{1}{r^{m}} \frac{\partial h}{\partial r}-r\left[g^{\prime \prime}+\frac{2(m+2)}{r} g^{\prime}-\frac{4}{r^{2}}(g+f)\right] \\
=-r^{2} f^{\prime \prime \prime}-[2 m+5] r f^{\prime \prime}-m(m+3) f^{\prime}, \\
+[m(m+3)+4] \frac{f+g}{r}-(m+1) g^{\prime}-r g^{\prime \prime} \\
=-r^{2} f^{\prime \prime \prime}-[2 m+5] r f^{\prime \prime}-[m(m+3)-2], \\
f^{\prime}+m(m+5) \frac{f+g}{r},
\end{gathered}
$$

where I have used

$g^{\prime}(r)=-\frac{2}{r}(f+g)$,

$r g^{\prime \prime}(r)=-2 f^{\prime}+\frac{6}{r}(f+g)$.

Inserting $f(r)$ and $g(r)$ expressions into the above equation yields $\mathcal{F}(r)$ so that in the end

$$
\begin{aligned}
\rho(r, \theta)= & {\left[2 \alpha r^{-(m+4)} \frac{m+3}{m+1}(m-1)\right.} \\
& \left.-\frac{2 \beta}{3}(m-1)(m+3)-m(m+5) \frac{2 \gamma}{r^{3}}\right] \cos \theta .
\end{aligned}
$$

\subsection{The pressure field}

The pressure is defined in Eq. (32) and $h(r)$ can now be computed.

\subsubsection{Case $m=-1$}

$h(r)=-\mu_{0} \frac{4}{r^{3}}\left(\alpha \ln r+\frac{\beta}{3} r^{3}+\gamma\right)=\frac{2}{r} \mu_{0} g(r)$

\subsubsection{Case $m \neq-1$}

$$
\begin{aligned}
h(r) & =-\mu(r) \frac{2(m+3)}{r^{3}}\left(-\frac{\alpha}{m+1} r^{-(m+1)}+\frac{\beta}{3} r^{3}+\gamma\right) \\
& =\frac{m+3}{r} \mu(r) g(r)
\end{aligned}
$$

\section{Additional measurements}

\subsection{Root mean square velocity}

Many benchmark studies (e.g., Blankenbach et al., 1989; Tosi et al., 2015) report the root mean square velocity quantity, defined as follows:

$$
v_{\mathrm{RMS}}=\sqrt{\frac{1}{V} \int_{V}|v|^{2} \mathrm{~d} V} .
$$

This is a convenient quantity as it captures (in an average sense) the nature of the velocity field in a single scalar value, which allows for an easy comparison either with a known analytical value or across multiple codes.

Since the velocity is known in all of the domain, it is a simple although tedious exercise to compute the RMS velocity. I find

$v_{\mathrm{RMS}}=\sqrt{\frac{4 \pi}{3 V}[B+4 A]}$,

with

$$
\begin{aligned}
A=\int_{R_{1}}^{R_{2}} f^{2} r^{2} \mathrm{~d} r, \\
B=\int_{R_{1}}^{R_{2}} g^{2} r^{2} \mathrm{~d} r .
\end{aligned}
$$

The values of $A$ and $B$ depend on the $f$ and $g$ function, so the distinction must once again be made between $m=-1$ and $m \neq-1$.

\subsubsection{Case $m=-1$}

$$
\begin{aligned}
& A=\left[-\frac{\alpha^{2}}{r}+\alpha \beta r^{2}+\frac{\beta^{2}}{5} r^{5}\right]_{R_{1}}^{R_{2}} \\
& B=4\left(B_{1}+B_{2}+B_{3}+B_{4}+B_{5}+B_{6}\right) \\
& B_{1}=\alpha^{2}\left[-\left(X^{2}+2 X+2\right) e^{-X}\right]_{\ln R_{1}}^{\ln R_{2}} \\
& B_{2}=\frac{2 \alpha \beta}{3}\left[\frac{1}{2} r^{2} \ln r-\frac{1}{4} r^{2}\right]_{R_{1}}^{R_{2}} \\
& B_{3}=2 \alpha \gamma\left[-X e^{-X}-e^{-X}\right]_{\ln R_{1}}^{\ln R_{2}} \\
& B_{4}=\frac{\beta^{2}}{45}\left[r^{5}\right]_{R_{1}}^{R_{2}} \\
& B_{5}=\frac{\beta \gamma}{3}\left[r^{2}\right]_{R_{1}}^{R_{2}} \\
& B_{6}=\gamma^{2}\left[-\frac{1}{r}\right]_{R_{1}}^{R_{2}}
\end{aligned}
$$

\subsubsection{Case $m \neq-1$}

$$
\begin{aligned}
& A=\left[-\frac{\alpha^{2}}{2 m+3} r^{-(2 m+3)}-\frac{2 \alpha \beta}{m-1} r^{-(m-1)}+\frac{\beta^{2}}{5} r^{5}\right]_{R_{1}}^{R_{2}} \\
& B=4\left(B_{1}+B_{2}+B_{3}-B_{4}-B_{5}+B_{6}\right) \\
& B_{1}=-\frac{\alpha^{2}}{(m+1)^{2}(2 m+3)}\left[r^{-(2 m+3)}\right]_{R_{1}}^{R_{2}} \\
& B_{2}=\frac{\beta^{2}}{45}\left[r^{5}\right]_{R_{1}}^{R_{2}}
\end{aligned}
$$




$$
\begin{aligned}
& B_{3}=-\gamma^{2}\left[\frac{1}{r}\right]_{R_{1}}^{R_{2}} \\
& B_{4}=-\frac{2 \alpha \beta}{3(m+1)(m-1)}\left[r^{-(m-1)}\right]_{R_{1}}^{R_{2}} \\
& B_{5}=-\frac{2 \alpha \gamma}{(m+1)(m+2)}\left[r^{-(m+2)}\right]_{R_{1}}^{R_{2}} \\
& B_{6}=\frac{\beta \gamma}{3}\left[r^{2}\right]_{R_{1}}^{R_{2}}
\end{aligned}
$$

\subsection{Radial averages}

The radial average of a quantity $\chi(r, \theta, \phi)$ is defined as follows:

$<\chi>_{R}=\frac{1}{4 \pi} \iint \chi(r, \theta, \phi) \sin \theta \mathrm{d} \theta \mathrm{d} \phi$,

and due to symmetry it is trivial to show that $\left\langle p>_{R}=0\right.$ and $<v_{r}>_{R}=0$. Likewise, one easily arrives at

$<v_{\theta}>_{R}=<v_{\phi}>_{R}=\frac{1}{4} f(r)$.

\subsection{Volume averages}

The volume average of a quantity $\chi(r, \theta, \phi)$ is defined as follows:

$$
<>_{V}=\frac{1}{V} \int_{V} \chi(r, \theta, \phi) \mathrm{d} V .
$$

Here again, $<p>_{V}=0$ and $<v_{r}>_{V}=0$, while

$$
\begin{aligned}
<v_{\theta}> & =<v_{\phi}>=\frac{\pi^{2}}{V} \int_{R_{1}}^{R_{2}} f(r) r^{2} \mathrm{~d} r \\
& = \begin{cases}\frac{\pi^{2}}{V}\left[\alpha r+\frac{\beta}{4} r^{4}\right]_{R_{1}}^{R_{2}} & (m=-1) \\
\frac{\pi^{2}}{V}\left[-\frac{\alpha}{m} r^{-m}+\frac{\beta}{4} r^{4}\right]_{R_{1}}^{R_{2}} & (m \neq-1)\end{cases}
\end{aligned}
$$

One can also look at the volume averages of the Cartesian coordinate components of the velocity.

$$
\begin{aligned}
& <v_{x}>=\frac{1}{V} \int_{V} v_{x}(r, \theta, \phi) \mathrm{d} V \\
& =\frac{1}{V} \int_{V}\left(\sin \theta \cos \phi v_{r}+\cos \theta \cos \phi v_{\theta}-\sin \phi v_{\phi}\right) \mathrm{d} V=0 \\
& <v_{y}>=\frac{1}{V} \int_{V} v_{y}(r, \theta, \phi) \mathrm{d} V \\
& =\frac{1}{V} \int_{V}\left(\sin \theta \sin \phi v_{r}+\cos \theta \sin \phi v_{\theta}+\cos \phi v_{\phi}\right) \mathrm{d} V=0 \\
& <v_{z}>=\frac{1}{V} \int_{V} v_{z}(r, \theta, \phi) \mathrm{d} V
\end{aligned}
$$

$$
\begin{aligned}
& =\frac{1}{V} \int_{V}\left(v_{r} \cos \theta-v_{\theta} \sin \theta\right) \mathrm{d} V \\
& =\frac{1}{V} \int_{V}\left(g(r) \cos ^{2} \theta-f(r) \sin ^{2} \theta\right) r^{2} \sin \theta \mathrm{d} r \mathrm{~d} \theta \mathrm{d} \phi \\
& =\frac{4 \pi}{3 V}\left[\int g(r) r^{2} \mathrm{~d} r-2 \int f(r) r^{2} \mathrm{~d} r\right]=0
\end{aligned}
$$

Note that $\left\langle v_{x}\right\rangle$ and $\left\langle v_{y}\right\rangle$ are zero because of symmetry $\left(\int_{0}^{2 \pi} \cos \phi \mathrm{d} \phi=\int_{0}^{2 \pi} \sin \phi \mathrm{d} \phi=0\right.$ ), while $<v_{z}>=0$ (for all values of $m$ ) after tedious calculations using the definitions of $\alpha$ and $\beta$.

\subsection{Surface averages}

The average of a quantity $\chi(r, \theta, \phi)$ on a surface of radius $R$ is simply the radial average function evaluated at a given radial distance $R$. Rather importantly, we have

$<p>_{R=R_{2}}=0$

i.e., the average pressure at the outside surface is zero.

\subsection{Moment of inertia}

Because of the expression of the density field, i.e., $\rho(r, \theta, \phi)=\mathcal{F}(r) \cos \theta$, it is trivial to show that the moment of inertia of the system with respect to the $x, y$, and $z$ axis are identically zero.

\subsection{Stress field}

Since the velocity and pressure fields are known, I can also compute an analytical expression for the stress field, and the stress tensor is given by

$$
\begin{aligned}
& \boldsymbol{\sigma}= \\
& \frac{\mu(r)}{r}\left(\begin{array}{ccc}
-((m+7) g+4 f) \cos \theta & \left(r f^{\prime}-f-g\right) \sin \theta & \left(r f^{\prime}-f\right) \sin \theta \\
\left(r f^{\prime}-f-g\right) \sin \theta & -((m+1) g-2 f) \cos \theta & 0 \\
\left(r f^{\prime}-f\right) \sin \theta & 0 & -((m+1) g-2 f) \cos \theta
\end{array}\right) .
\end{aligned}
$$

\section{Implementation and results}

As mentioned earlier, this flow solution was designed with a geodynamics application in mind. It has therefore been implemented in the state-of-the-art open-source code ASPECT ${ }^{1}$ (Kronbichler et al., 2012; Heister et al., 2017) and in the ELEFANT $^{2}$ code (Thieulot, 2014; Tosi et al., 2015; Lavecchia et al., 2017). Both codes solve the incompressible flow Stokes equations in spherical shell domains but use Cartesian coordinates.

\footnotetext{
${ }^{1}$ https://aspect.dealii.org/

${ }^{2} \mathrm{http}: / /$ cedricthieulot.net/elefant.html
} 

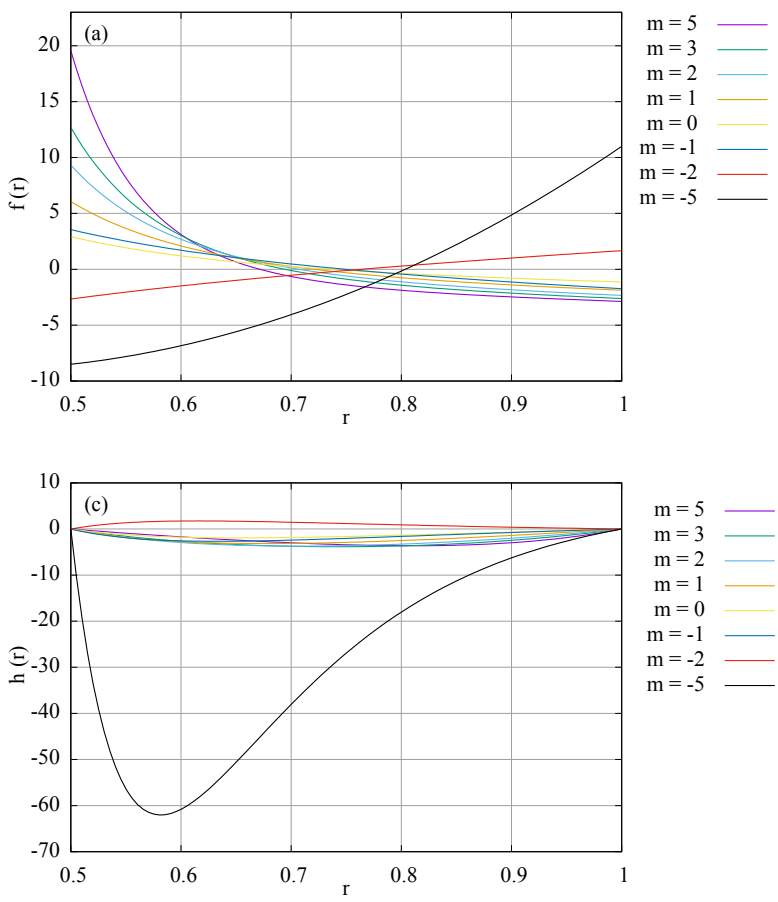
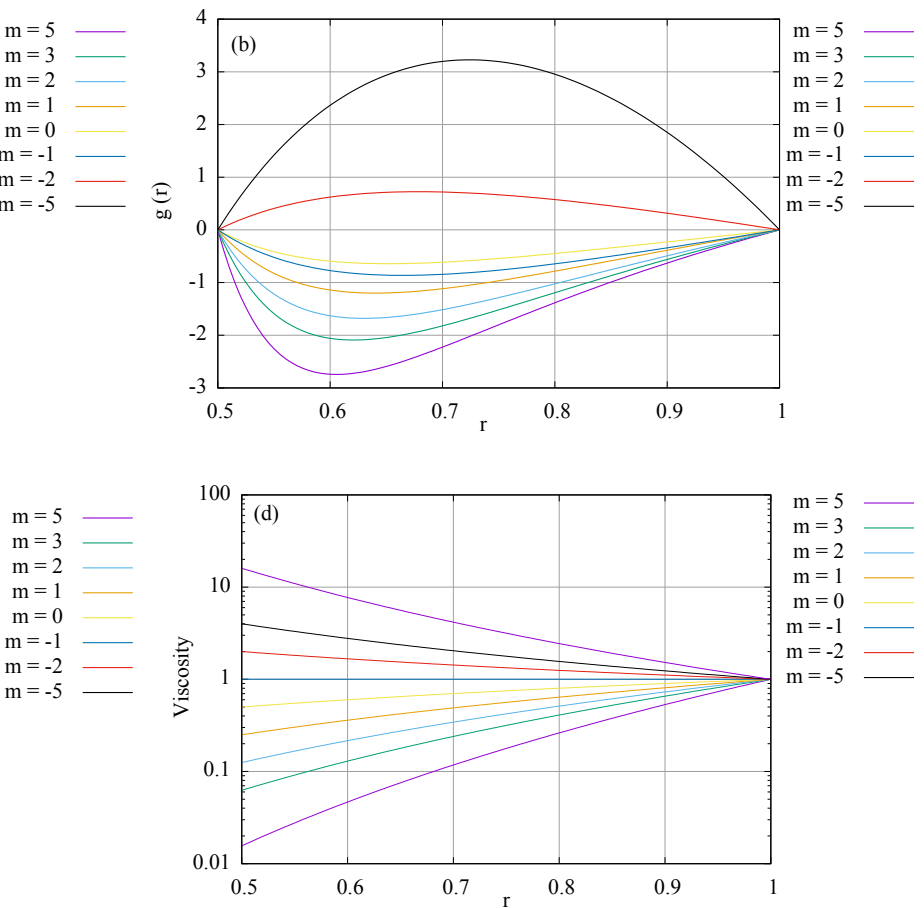

Figure 1. Functions $f(r)$ (a), $g(r)(\mathbf{b}), h(r)(\mathbf{c})$, and viscosity profile $\mu(r)(\mathbf{d})$ as a function of $r \in[0.5: 1]$ for various values of $m$.

\subsection{ASPECT}

ASPECT is a finite-element code intended to solve the equations that describe thermally driven convection with a focus on doing so in the context of convection in Earth's mantle. The default element type $Q_{2} Q_{1}$ (quadratic velocity, linear pressure) has been used in this work, but since ASPECT is based on the deal.ii library (Bangerth et al., 2007, 2016), one can easily change the element type from the ASCII input file (".prm" file), and the $Q_{1} P_{0}$ (linear velocity, constant pressure) was also used (the same element is used in the CITCOM code; Zhong et al., 2008).

I make use of the plugin architecture of the code which allows users and developers to easily add or switch between already-implemented features. The flow velocity and pressure solutions, as well as the viscosity and body force expressions, are all encapsulated in a single piece of code alongside an ASCII input file in which resolution, element type, boundary conditions, and other parameters are set. This benchmark is now part of the mainline since version 2.0.0-pre (see ASPECT manual).

\subsection{ELEFANT}

ELEFANT borrows largely from the FANTOM code (Thieulot, 2011), but it also brings a number of critical improvements compared to its predecessor, such as spherical shell geometry and the use of a preconditioned conjugate gradient scheme for both inner and outer iterations (Braess, 2007;
Elman, 1996). It is a finite-element code based on $Q_{1} P_{0}$ elements, which is developed and maintained by the author. It has successfully been benchmarked against a wide range of analytical problems and also against other codes (including ASPECT) in the case of visco-plastic thermal convection (Tosi et al., 2015).

\subsection{Setup}

In what follows, I set the inner and outer radii to $R_{1}=0.5$ and $R_{2}=1$, respectively. The functions $f(r), g(r), h(r)$, and the viscosity function are shown in Fig. 1.

The ASPECT computational grid consists of 12 blocks making up a hollow sphere (Zhong et al., 2000), with each block subdivided into $\left(2^{n}\right)^{3}$ elements, where $n=2,3, \ldots$. Since elements vary in size in the radial direction, I have chosen to report the average element size in convergence plots, and it is computed as follows:

$<h>=\left(\frac{V}{N_{\mathrm{el}}}\right)^{1 / 3}$,

where $V$ is the volume of the domain and $N_{\mathrm{el}}$ is the total number of elements. The ELEFANT mesh is also based on the same 12 blocks, but each block can be subdivided into $n e l^{3}$ elements, where nel is a positive integer that is not bound to be a power of 2 .

The analytical velocity solution is prescribed on both internal $\left(r=R_{1}\right)$ and external $\left(r=R_{2}\right)$ boundaries. Given these boundary conditions, the pressure is determined up to a con- 

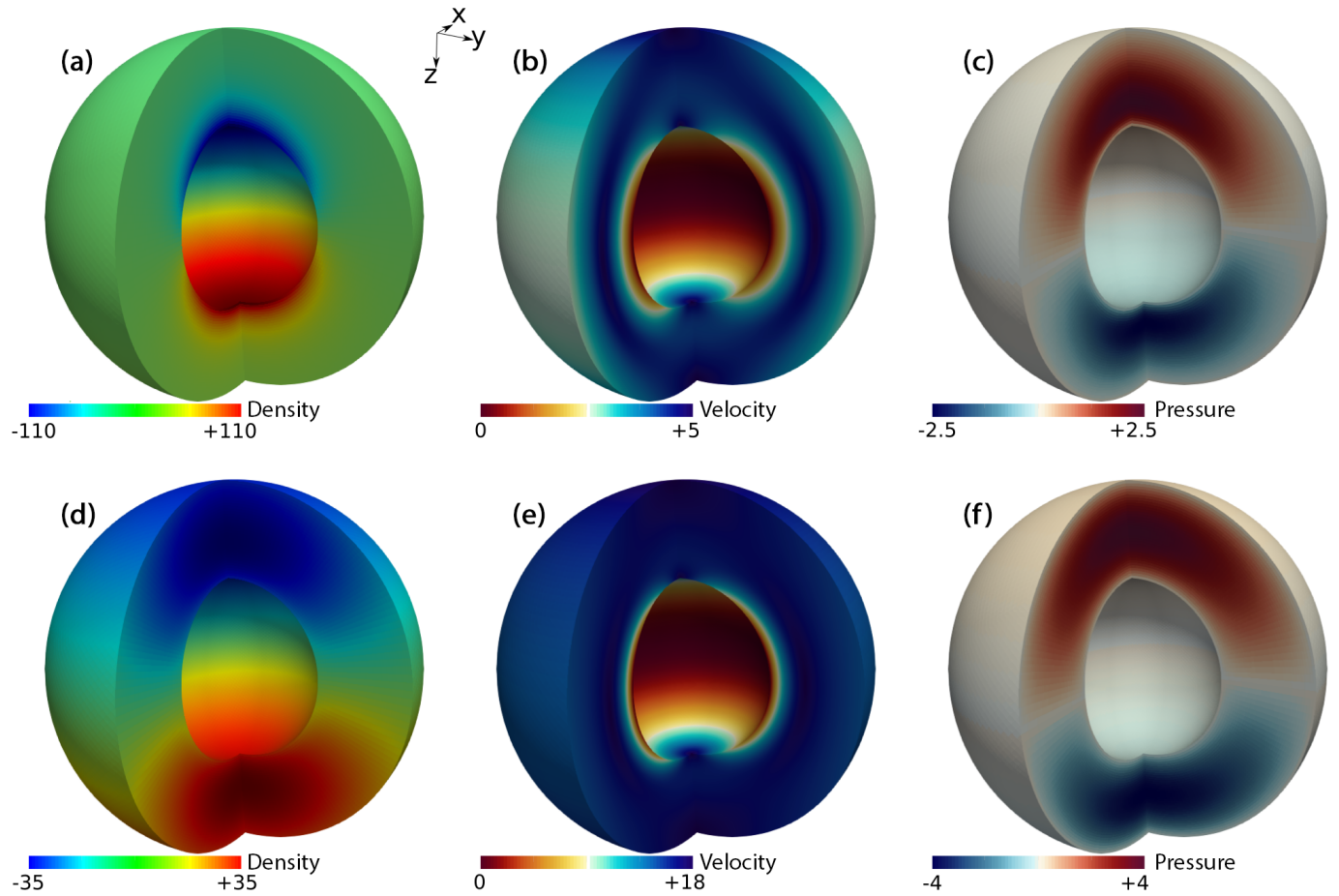

Figure 2. (a-c) Analytical solution for $m=-1$ (constant viscosity); (d-f) solution for $m=3$.

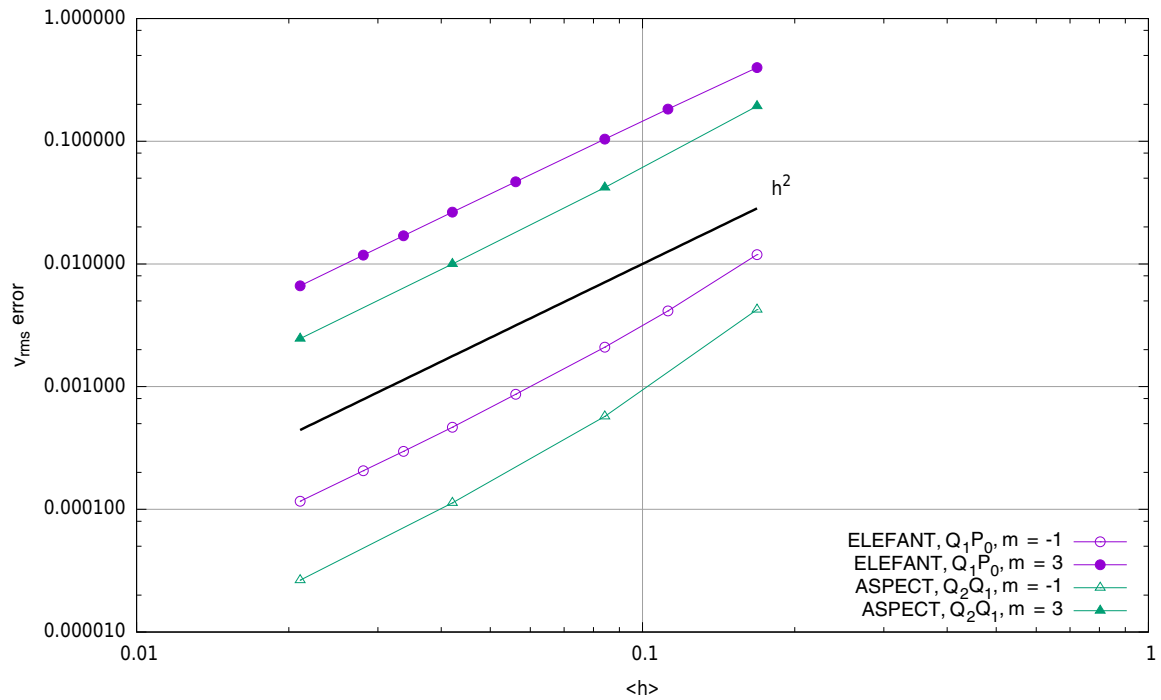

Figure 3. Root mean square velocity as a function of the average element size for both codes.

stant. Both codes allow for a surface normalization (the average pressure along the surface has a prescribed value, in this case zero) and a volume normalization (the average pressure over the whole volume has a prescribed zero value). Since I have shown above that both are identically null for the pressure field, the choice of pressure normalization does not matter. Also, the boundary conditions preclude the presence of a pure rotational mode of numerical origin (Zhong et al.,
2008). Both codes were run for values of $m=-1$ (constant viscosity) and $m=3$ (viscosity varies by a factor 16 from the inside to the outside). The density, velocity, and pressure fields for both cases are shown in Fig. 2.

Figure 3 shows the relative root mean square velocity error as a function of the (average) element size for both codes and both $m$ values. The error is found to quadratically decrease with resolution for both codes. Likewise, the $L_{2}$-norm of the 

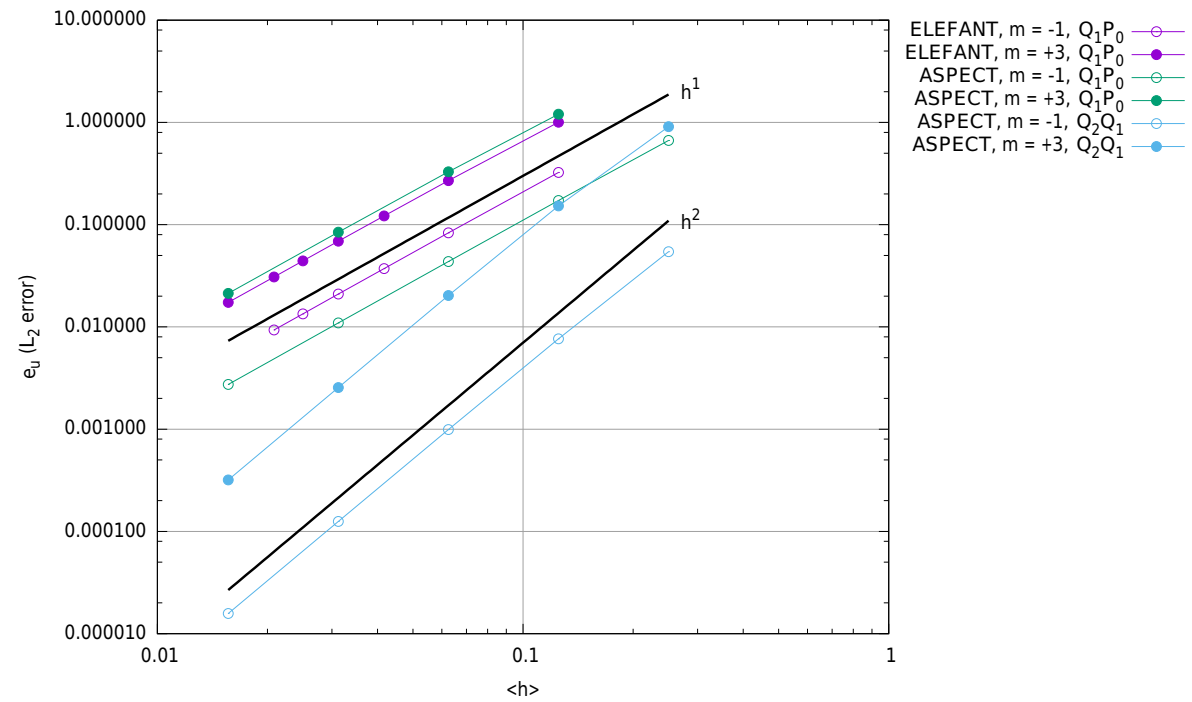

Figure 4. Velocity $L_{2}$-norm error vs. average resolution for ELEFANT and ASPECT.

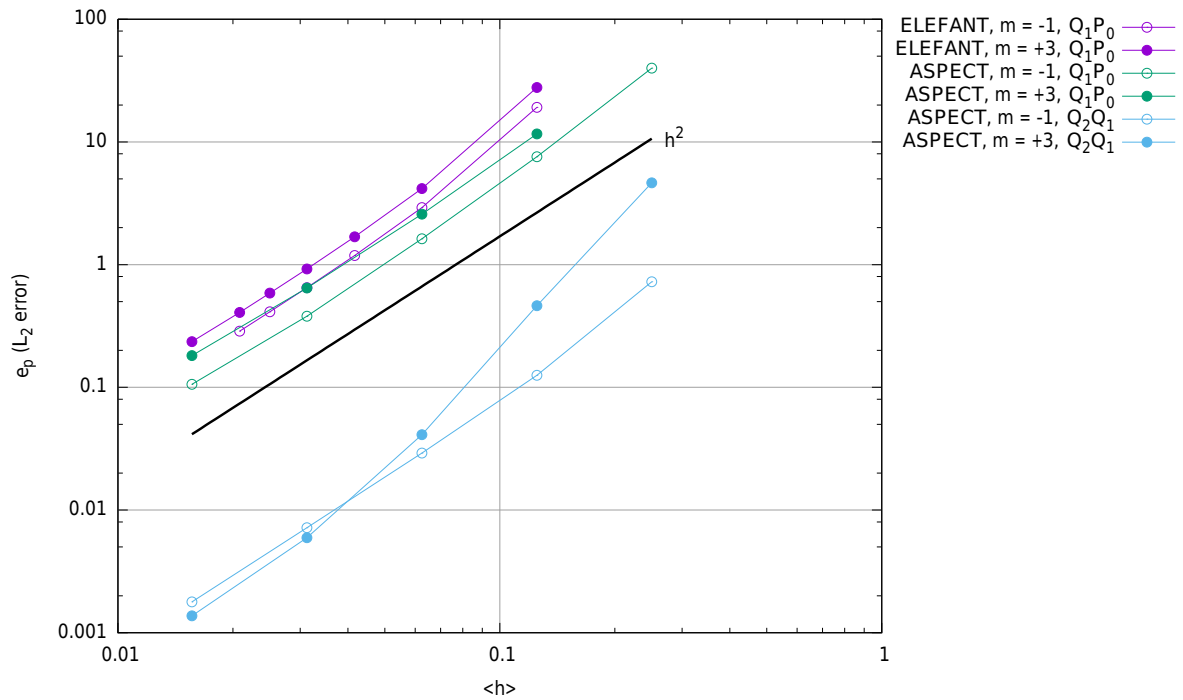

Figure 5. Pressure $L_{2}$-norm error vs. average resolution for ELEFANT and ASPECT.

velocity error is found to decrease with resolution, linearly for $Q_{1} P_{0}$ elements and quadratically for $Q_{2} Q_{1}$, as shown in Fig. 4. Looking at the pressure error convergence, it is found to decrease quadratically with the resolution for both types of elements, as shown in Fig. 5. ELEFANT routinely outputs all three average quantities $\langle u\rangle,\langle v\rangle$, and $\langle w\rangle$. All three values were found to be zero within machine precision (oscillating around $10^{-15}$ ).

\section{Conclusions}

I have derived in this paper a family of analytical solutions to incompressible Stokes flow in a spherical shell under a few assumptions, such as tangential velocity on the boundaries and a radial viscous profile. The velocity, pressure, density, and viscous fields that satisfy the flow equations at every point in space are then used to benchmark two multipurpose geodynamics codes. The $L_{2}$-norms of the velocity and pressure errors were reported and shown to decrease when the resolution is increased. Furthermore, various analytical expressions for flow averages were derived, and it was shown that the computed solutions converged to these expected values.

A number of previous studies (Popov et al., 2014) use an exponential viscosity of the form $\mu(r)=\mu_{0} \exp (\boldsymbol{\alpha} \cdot \boldsymbol{r})$, where $\alpha$ is a parameter controlling the amplitude of the viscosity 
variations in the system. This approach was tried during the preparation of the paper, but Eq. (35) then becomes

$r^{2} f^{\prime \prime}+(2+m r) r f^{\prime}-(2+m r) f=0$.

Although this equation can be solved, the form of the solution $f(r)$ involves the exponential integral function $\operatorname{Ei}(r)=$ $-\int_{-r}^{\infty} e^{-t} / t \mathrm{~d} t$, which would (a) render the derivations of $g(r)$ and all subsequent quantities very cumbersome and (b) make the solution only semi-analytical. This approach was then abandoned.

Looking at the pressure equation, or rather at Fig. 1c, we see that the pressure is zero for $r=R_{1}$ and $r=R_{2}$. One simple interpretation is that the pressure in this work should be interpreted as an over pressure with regards to a background lithostatic pressure. Likewise, the (complex) density profiles have to be interpreted as density variations with regards to a background density profile corresponding to the above-mentioned lithostatic pressure.

Finally, it must be mentioned that the driving density field and flow solution both consist azimuthally of spherical harmonic degree 1 and order 0 combined with an azimuthally constant viscosity. Presumably, the presented solutions are part of a family of solutions that could also be derived for other spherical harmonics.

Data availability. This benchmark has been part of the opensource ASPECT code since version 2.0.0-pre (see ASPECT manual; Bangerth et al., 2017).

Author contributions. All analytical derivations, equations, implementation, and numerical simulations have been carried out by the author.

Competing interests. The author declares no conflict of interest.

Acknowledgements. The author acknowledges stimulating discussions with Elbridge Gerry Puckett in the early stages of this work and technical help from Anne Glerum and Menno Fraters with all things ASPECT. Early reviews by Matt Weller and additional HPC runs by Harsha Lokavarapu are gratefully acknowledged.

Edited by: Taras Gerya

Reviewed by: two anonymous referees

\section{References}

Arrial, P.-A., Flyer, N., Wright, G. B., and Kellogg, L. H.: On the sensitivity of 3-D thermal convection codes to numerical discretization: a model intercomparison, Geosci. Model Dev., 7, 2065-2076, https://doi.org/10.5194/gmd-7-2065-2014, 2014.
Bangerth, W., Hartmann, R., and Kanschat, G.: deal.II - a general purpose object oriented finite element library, ACM T. Math. Software, 33, https://doi.org/10.1145/1268776.1268779, 2007.

Bangerth, W., Davydov, D., Heister, T., Heltai, L., Kanschat, G., Kronbichler, M., Maier, M., Turcksin, B., and Wells, D.: The deal.ii library, version 8.4, J. Numer. Math., 24, https://doi.org/10.1515/jnma-2016-1045, 2016.

Bangerth, W., Dannberg, J., Gassmöller, R., Heister, T., and others: ASPECT: Advanced Solver for Problems in Earth's ConvecTion, User Manual, https://doi.org/10.6084/m9.figshare.4865333, 2017.

Bercovici, D., Schubert, G., Glatzmaier, G., and Zebib, A.: Threedimensional thermal convection in a spherical shell, J. Fluid Mech., 206, 75-104, 1989.

Blankenbach, B., Busse, F., Christensen, U., Cserepes, L., Gunkel, D., Hansen, U., Harder, H., Jarvis, G., Koch, M., Marquart, G., Moore, D., Olson, P., Schmeling, H., and Schnaubelt, T.: A benchmark comparison for mantle convection codes, Geophys. J. Int., 98, 23-38, 1989.

Blinova, I., Makeev, I., and Popov, I.: Benchmark solutions for Stokes flows in cyclindrical and spherical geometry, Bulletin of the Transilvania University of Brasov, 9, 58-63, 2016.

Bower, D., Gurnis, M., and Flament, N.: Assimilating lithosphere and slab history in 4-D Earth models, Phys. Earth. Planet. In., 238, 8-22, 2015.

Braess, D.: Finite Elements, Cambridge, UK, 2007.

Bull, A., Domeier, M., and Torsvik, T.: The effect of plate motion history on the longevity of deep mantle heterogeneities, Earth Planet. Sc. Lett., 401, 172-182, 2014.

Burstedde, C., Stadler, G., Alisic, L., Wilcox, L., Tan, E., Gurnis, M., and Ghattas, O.: Large-scale adaptive mantle convection simulation, Geophys. J. Int., 192, 889-906, 2013.

Busse, F.: Patterns of convection in spherical shells, J. Fluid Mech., 72, 67-85, 1975.

Busse, F. and Riahi, N.: Patterns of convection in spherical shells Part 2, J. Fluid Mech., 123, 283-301, 1982.

Choblet, G., Čadek, O., Couturier, F., and Dumoulin, C.: OEDIPUS: a new tool to study the dynamics of planetary interiors, Geophy. J. Int., 170, 9-30, 2007.

Crameri, F. and Tackley, P.: Subduction initiation from a stagnant lid and global overturn: new insights from numerical models with a free surface, Progress in Earth and Planetary Science, 3, https://doi.org/10.1186/s40645-016-0103-8, 2016.

Dannberg, J. and Heister, T.: Compressible magma/mantle dynamics: 3-D, adaptive simulations in ASPECT, Geophy. J. Int., 207, 1343-1366, 2016.

Davies, D. R., Davies, J. H., Bollada, P. C., Hassan, O., Morgan, K., and Nithiarasu, P.: A hierarchical mesh refinement technique for global 3-D spherical mantle convection modelling, Geosci. Model Dev., 6, 1095-1107, https://doi.org/10.5194/gmd-6-10952013, 2013.

Elman, H.: Multigrid and Krylov subspace methods for the discrete Stokes equations, Int. J. Numer. Meth. Fl., 22, 755-770, 1996.

Glatzmaier, G.: Numerical simulations of mantle convection: Timedependent, three-dimensional, compressible, spherical shell, Geophys. Astro. Fluid, 43, 223-264, 1988.

Hager, B. and O'Connell, R.: A simple global model of plate dynamics and mantle convection, J. Geophys. Res., 86, 4843-4867, 1981. 
Heister, T., Dannberg, J., Gassmöller, R., and Bangerth, W.: High Accuracy Mantle Convection Simulation through Modern Numerical Methods. II: Realistic Models and Problems, Geophy. J. Int., 210, 833-851, 2017.

Iwase, Y.: Three-dimensional infinite Prandtl number convection in a spherical shell with temperature-dependent viscosity, J. Geomagn. Geoelectr., 48, 1499-1514, 1996.

Kageyama, A. and Sato, T.: "Yin-Yang grid": An overset grid in spherical geometry, Geochem. Geophy. Geosys., 5, Q09005, https://doi.org/10.1029/2004GC000734, 2004.

Kronbichler, M., Heister, T., and Bangerth, W.: High accuracy mantle convection simulation through modern numerical methods, Geophy. J. Int., 191, 12-29, 2012.

Lavecchia, A., Thieulot, C., Beekman, F., Cloetingh, S., and Clark, S.: Lithosphere erosion and continental breakup: Interaction of extension, plume upwelling and melting, Earth Planet. Sc. Lett., 467, 89-98, 2017.

Lay, T. and Garnero, E.: Deep Mantle Seismic Modeling and Imaging, Annu. Rev. Earth Pl. Sc., 39, 91-123, 2011.

Machetel, P., Rabinowitz, M., and Bernardet, P.: Three-dimensional convection in spherical shells, Geophys. Astro. Fluid, 37, 57-84, 1986.

Popov, I. Yu., Lobanov, I. S., Popov, S. I., Popov, A. I., and Gerya, T. V.: Practical analytical solutions for benchmarking of 2-D and 3-D geodynamic Stokes problems with variable viscosity, Solid Earth, 5, 461-476, https://doi.org/10.5194/se-5-461-2014, 2014.

Ratcliff, J., Schubert, G., and Zebib, A.: Steady tetrahedral and cubic patterns of spherical shell convection with temperaturedependent viscosity, J. Geophys. Res., 101, 25473-25484, 1996.

Richards, M. and Hager, B.: Geoid anomalies in a dynamic Earth, J. Geophys. Res., 89, 5987-6002, 1984.

Richards, M., Yang, W.-S., Baumgardner, J., and Bunge, H.-P.: Role of a low-viscosity zone in stabilizing plate tectonics: Implications for comparative terrestrial planetology, Geochem. Geophys. Geosy., 2, 1026, https://doi.org/10.1029/2000GC000115, 2001.

Schubert, G., Turcotte, D., and Olson, P.: Mantle Convection in the Earth and Planets, Cambridge University Press, Cambridge, UK, 2001.

Stemmer, K., Harder, H., and Hansen, U.: A new method to simulate convection with strongly temperature- and pressure-dependent viscosity in a spherical shell: Applications to the Earth's mantle, Phys. Earth Planet. In., 157, 223-249, 2006.

Tabata, M. and Suzuki, A.: A stabilized finite element method for the Rayleigh-Bénard equations with infinite Prandtl number in a spherical shell, Comput. Method. Appl. M., 190, 387-402, 2000.

Tackley, P.: Modelling compressible mantle convection with large viscosity contrasts in a three-dimensional spherical shell using the yin-yang grid, Phys. Earth Planet. In., 171, 7-18, 2008.

Tackley, P.: Dynamics and evolution of the deep mantle resulting from thermal, chemical, phase and melting effects, Earth-Sci. Rev., 110, 1-25, 2012.
Thieulot, C.: FANTOM: two- and three-dimensional numerical modelling of creeping flows for the solution of geological problems, Phys. Earth Planet. Int., 188, 47-68, 2011.

Thieulot, C.: ELEFANT: a user-friendly multipurpose geodynamics code, Solid Earth Discuss., https://doi.org/10.5194/sed-6-19492014, in review, 2014.

Tosi, N. and Martinec, Z.: Semi-analytical solution for viscous Stokes flow in two eccentrically nested spheres, Geophys. J. Int., 170, 1015-1030, 2007.

Tosi, N., Stein, C., Noack, L., Huettig, C., Maierova, P., Samuel, H., Davies, D., Wilson, C., Kramer, S., Thieulot, C., Glerum, A., Fraters, M., Spakman, W., Rozel, A., and Tackley, P.: A community benchmark for viscoplastic thermal convection in a 2-D square box, Geochem. Geophy. Geosy., 16, 2175-2196, https://doi.org/10.1002/2015GC005807, 2015.

van Heck, H. J., Davies, J. H., Elliott, T., and Porcelli, D.: Globalscale modelling of melting and isotopic evolution of Earth's mantle: melting modules for TERRA, Geosci. Model Dev., 9, 13991411, https://doi.org/10.5194/gmd-9-1399-2016, 2016.

van Hinsbergen, D., Steinberger, B., Doubrovine, P., and Gassmöller, R.: Acceleration and deceleration of India-Asia convergence since the Cretaceous: Roles of mantle plumes and continental collision, J. Geophys. Res., 116, B06101, https://doi.org/10.1029/2010JB008051, 2011.

van Keken, P., Hauri, E., and Ballentine, C.: Mantle mixing: the generation, preservation and destruction of chemical heterogeneity, Annu. Rev. Earth Pl. Sc, 30, 493-525, 2002.

Yoshida, M. and Kageyama, A.: Application of the YinYang grid to a thermal convection of a Boussinesq fluid with infinite Prandtl number in a three-dimensional spherical shell, Geophys. Res. Lett., 31, L12609, https://doi.org/10.1029/2004GL019970, 2004.

Zhang, S. and Christensen, U.: Some effects of lateral viscosity variations on geoid and surface velocities induced by density anomalies in the mantle, Geophy. J. Int., 114, 531-547, 1993.

Zhang, S. and Yuen, D.: The influences of lower mantle viscosity stratification on 3D spherical-shell mantle convection, Earth Planet. Sc. Lett., 132, 157-166, 1995.

Zhong, S.: Analytic solutions for Stokes' flow with lateral variations in viscosity, Geophys. J. Int., 124, 18-28, 1996.

Zhong, S., Zuber, M., Moresi, L., and Gurnis, M.: The role of temperature-dependent viscosity and surface plates in spherical shell models of mantle convection, J. Geophys. Res., 105, 11063-11082, 2000.

Zhong, S., McNamara, A., Tan, E., Moresi, L., and Gurnis, M.: A benchmark study on mantle convection in a 3-D spherical shell using CITCOMS, Geochem. Geophy. Geosy., 9, Q10017, https://doi.org/10.1029/2008GC002048, 2008. 